BRIEF COMMUNICATION

\title{
The retina visual cycle is driven by cis retinol oxidation in the outer segments of cones
}

\author{
SHINYA SATO, ${ }^{1}$ RIKARD FREDERIKSEN,${ }^{2}$ M. CARTER CORNWALL,${ }^{2}$ AND \\ VLADIMIR J. KEFALOV ${ }^{1}$ \\ ${ }^{1}$ Department of Ophthalmology and Visual Sciences, Washington University School of Medicine, Saint Louis, Missouri 63110 \\ ${ }^{2}$ Department of Physiology and Biophysics, Boston University School of Medicine, Boston, MA 02118 \\ (ReCeIved December 8, 2016; ACCEPTED January 9, 2017)
}

\begin{abstract}
Vertebrate rod and cone photoreceptors require continuous supply of chromophore for regenerating their visual pigments after photoactivation. Cones, which mediate our daytime vision, demand a particularly rapid supply of 11-cis retinal chromophore in order to maintain their function in bright light. An important contribution to this process is thought to be the chromophore precursor 11-cis retinol, which is supplied to cones from Müller cells in the retina and subsequently oxidized to 11-cis retinal as part of the retina visual cycle. However, the molecular identity of the cis retinol oxidase in cones remains unclear. Here, as a first step in characterizing this enzymatic reaction, we sought to determine the subcellular localization of this activity in salamander red cones. We found that the onset of dark adaptation of isolated salamander red cones was substantially faster when exposing directly their outer $v s$. their inner segment to 9-cis retinol, an analogue of 11-cis retinol. In contrast, this difference was not observed when treating the outer $v s$. inner segment with 9-cis retinal, a chromophore analogue which can directly support pigment regeneration. These results suggest, surprisingly, that the cis-retinol oxidation occurs in the outer segments of cone photoreceptors. Confirming this notion, pigment regeneration with exogenously added 9-cis retinol was directly observed in the truncated outer segments of cones, but not in rods. We conclude that the enzymatic machinery required for the oxidation of recycled cis retinol as part of the retina visual cycle is present in the outer segments of cones.
\end{abstract}

Keywords: Cone photoreceptors, Pigment regeneration, Dark adaptation, Retinol dehydrogenase, Visual cycle

\section{Introduction}

The vertebrate visual pigment, the light receptor molecule of rod and cone photoreceptor cells, is composed of a protein moiety, opsin, and chromophore, typically 11-cis retinal. Upon absorbing light, 11-cis retinal is photoisomerized to its all-trans form to activate the pigment before eventually dissociating from opsin [reviewed in (Ebrey \& Koutalos, 2001; Imamoto \& Shichida, 2014)]. The continuous function of photoreceptor cells requires regeneration of their visual pigments with newly synthesized 11-cis retinal. In vertebrate eyes, there are two metabolic pathways for 11-cis retinal synthesis: the retinal pigment epithelium (RPE) visual cycle and the retina visual cycle (Wang \& Kefalov, 2011; Saari, 2012; Tang et al., 2013; Wright et al., 2015). The RPE visual cycle is the canonical pathway in which 11-cis retinal is synthesized in the RPE cells and supplied to both rods and cones. The retina visual cycle, on the other hand, is a recently discovered pathway in which 11-cis retinol is synthesized in Müller cells, supplied to cones, and then oxidized there to 11-cis retinal. This cis retinol oxidation

Address correspondence to: Vladimir J. Kefalov, Ph.D, Department of Ophthalmology and Visual Sciences, Washington University in Saint Louis, 660 S. Euclid Avenue, Saint Louis, MO 63110. E-mail: kefalov@wustl.edu activity is cone-specific (Jones et al., 1989; Miyazono et al., 2008; Ala-Laurila et al., 2009; Sato \& Kefalov, 2016). As a result, the retina visual cycle can supply chromophore exclusively to cones, enhancing in that way their fast dark adaptation and their function in bright light (Mata et al., 2002; Kolesnikov et al., 2011; Wang et al., 2014). However, little is known about the molecular mechanisms of the retina visual cycle. Although recent studies have identified several putative components of the pathway for 11-cis retinoid synthesis in Müller cells (Kaylor et al., 2013; Kaylor et al., 2014; Xue et al., 2015), the enzyme(s) responsible for the cis retinol oxidation in cones is still unknown.

Two hypotheses about this cone-specific reaction have been recently proposed. One suggestion is that the oxidation of $\mathrm{cis}$ retinol is carried out in the outer segments of cones by retinol dehydrogenase 8 (RDH8). This idea is based on the observation that deletion of RDH8 blocks the cis retinol-dependent sensitivity recovery in mouse M-cones (Kolesnikov et al., 2015). However, RDH8 is expressed in the outer segments of both rods and cones (Maeda et al., 2005; Miyazono et al., 2008) and is thought to be primarily a reductase of all-trans retinal rather than an oxidase of cis retinol (Rattner et al., 2000). The alternative hypothesis is that the oxidation of cis retinol takes place in cone inner segments and is carried out by RDH13-like (RDH13L) and its homologs (Sato et al., 2015). 
RDH13L is a cone-specific enzyme identified from purified carp cone inner segment membranes. It oxidizes cis retinol with concomitant reduction of $\mathrm{NADP}^{+}$or hydrophobic aldehydes. However, $\mathrm{RDH} 13 \mathrm{~L}$ is found only in fish and is, therefore, not a component of the mammalian retina visual cycle. Although it's functional homolog, RDH14 (Sato et al., 2015), is suggested to be expressed in mouse cones (Kanan et al., 2008), its role in the cone-specific oxidation of cis retinol has not been examined.

Here, as a way of addressing this issue, we sought to identify the cellular compartment of salamander red cones where $c$ is retinol oxidation takes place. The salamander retina has a high percentage of cones $(\sim 40 \%)$ (Sherry et al., 1998) and its photoreceptors are $>5$ times larger in width than those of the mouse retina [see for instance (Koutalos \& Cornwall, 2010)]. These features allowed us targeted application of cis retinal or cis retinol selectively to the inner or outer segment of individual cones in an effort to determine the subcellular localization of the cis retinol oxidation activity in cones.

\section{Materials and methods}

\section{Single-cell suction recordings}

All experiments were performed in accordance with protocols approved by the Washington University Animal Studies Committee and the Institutional Animal Care and Use Committee of Boston University School of Medicine, following the Guide for the Care and Use of Laboratory Animals and the Animal Welfare Act. Larval tiger salamanders (Ambystoma tigrinum, Charles D. Sullivan Co. Inc., Nashville, TN) were maintained on a 12-h day-night cycle and were dark-adapted overnight before use. Single-cell recordings from salamander cones were carried out as previously described (Kefalov et al., 2001). For the cone dark-adaptation experiments, the inner or outer segment of a red cone was first drawn in the suction pipette and after measuring its dark current and sensitivity, the cell was exposed to brief bright $505 \mathrm{~nm}$ light estimated to bleach $>99 \%$ of its visual pigment. Bleaching light duration was determined from the relation $F=1-\exp (-I P t)$, where $F$ is the fraction of pigment to be bleached $(>0.99), t$ is the duration of light exposure $(>18 \mathrm{~s}), I$ is the light intensity $\left(1.2 \times 10^{8}\right.$ photons $\left.\cdot \mu \mathrm{m}^{-2} \cdot \mathrm{sec}^{-1}\right)$, and $P$ is the photosensitivity of salamander red cones at $505 \mathrm{~nm}$ $\left(2.3 \times 10^{-9} \mu \mathrm{m}^{2}\right)$, estimated from the action spectrum of salamander red cones (Makino \& Dodd, 1996; Sato \& Kefalov, 2016) and the reported photosensitivity value at the wavelength of peak absorbance $\left(6.0 \times 10^{-9} \mu \mathrm{m}^{2}\right)$ (Jones et al., 1993). Following the bleach, the cell was incubated in darkness for 20 min to allow the photoactivated visual pigments to decay to free opsin. The bleach-adapted cell was then treated with $800 \mu \mathrm{l}$ of $125 \mu \mathrm{M}$ retinoid solution which was added to the recording chamber. The retinoid solution was prepared as follows: $300 \mu \mathrm{g}$ of dry retinoid (9-cis retinol: Santa Cruz Biotechnology, Inc., Santa Cruz, CA; 9-cis retinal: Sigma-Aldrich, Inc., Saint Louis, MO) was dissolved into $11 \mu \mathrm{l}$ ethanol and kept on ice until used. Just before adding to the recording chamber, $1 \mu \mathrm{l}$ of the ethanolic retinoid solution was mixed with $100 \mu$ l of Ringer's solution first and then additional $700 \mu$ l of Ringer's solution was added gradually to obtain $125 \mu \mathrm{M}$ retinoid solution. The retinoid solution thus prepared was added into the recording chamber containing $\sim 2 \mathrm{ml}$ of perfusion solution (i.e., final concentration: $\sim 40 \mu \mathrm{M}$ ) using a pipette with a wide-bore tip. To optimize the delivery of retinoids to the cone held in the suction electrode, the solution in the chamber was stirred by flushing some of it in and out of the pipette 3 times. As the solution was applied to the bath, only the segment of the cell exposed to it and not drawn in the suction electrode was treated with the retinoid (Jin et al., 1994). To monitor the recovery of cone sensitivity following the application of the retinoid, dim flash responses (with amplitudes below $30 \%$ of the maximum recorded from each cone) were recorded every $1 \mathrm{~min}$ for 9-cis retinal-treated cones or every $2 \mathrm{~min}$ for 9-cis retinol-treated cones, up to $20 \mathrm{~min}$ after the addition of retinoid solution. Flash sensitivities were determined as the ratio of dim flash response amplitude and flash intensity. Electrophysiology data were analyzed using pClamp 9 (Molecular Devices, LLC, Sunnyvale, CA) and Origin 7.5 (OriginLab, Northampton, MA).

\section{Microspectrophotometry measurements}

The microspectrophotometry measurements were performed on a previously described instrument (Frederiksen et al., 2012). At the beginning of an MSP experiment, a dark-adapted retina was chopped and triturated. Solution containing isolated intact cells and outer segments was placed on a quartz cover-slip window located in the bottom of a $2 \mathrm{~mm}$-deep Plexiglass recording chamber. The tissue was allowed to settle and adhere to the quartz window for up to 10 min to minimize movement of the cells, after which the tissue in the chamber was superfused at a rate of $4 \mathrm{ml} / \mathrm{min}$ with Ringer's solution identical to that used in the electrophysiological experiments. At this time, an isolated intact photoreceptor cell or outer segments were identified from their projected image on the screen of the infrared video system. A baseline absorbance spectrum was measured in an area adjacent to the selected outer segment. Following this measurement, the outer segment was oriented in the beam path with the polarization of the incident measuring beam parallel to the plane of the disks (rods) or invaginations (cones), and absorbance spectra in this area were measured in steps of $2 \mathrm{~nm}$ step width. Generally, ten complete sample scans and ten baseline scans were averaged to increase the signal-to-noise ratio of a measured spectrum. The absorbance spectrum was calculated using Beers' Law according to the relation; OD $=\log _{10}\left(I_{\mathrm{o}} / I_{\mathrm{t}}\right)$, where OD is optical density or absorbance, $I_{\mathrm{o}}$ is the light transmitted through a cell-free space adjacent to the outer segment, and $I_{\mathrm{t}}$ is the light transmitted through the outer segment.

Dark-adapted spectra of the visual pigment were measured in this way from rod and cone outer segments. Once the dark-adapted spectral absorbance was established, the pigment was bleached (>99\%) using a calibrated $\left(505 \mathrm{~nm} ; 1.3 \times 10^{7}\right.$ photons $\left.\cdot \mu \mathrm{m}^{-2} \cdot \mathrm{sec}^{-1}\right)$ LED light source. Bleaching photoproducts were monitored periodically and were allowed to decay to undetectable levels. This period was $10 \mathrm{~min}$ for cone outer segments, and about $60 \mathrm{~min}$ for rods. Following this, we measured the bleached absorbance spectrum. Next, we treated the preparation with $1 \mathrm{ml}$ of $40 \mu \mathrm{M}$ 9-cis retinol for $10 \mathrm{~min}$. Spectral absorbance was measured and the pigment regeneration was monitored until no further change in spectral absorbance could be detected. Then the regenerated pigment (cones) was bleached once more to confirm that it was photosensitive. If no pigment was regenerated (rods) the preparation was treated with $1 \mathrm{ml}$ of $40 \mu \mathrm{M} 11$-cis retinal for $10 \mathrm{~min}$ and allowed to regenerate visual pigment.

\section{Statistics}

A two-tailed unpaired Student's $t$-test was used to test for the significance of difference in the mean values of two sample groups. $P$-values of $<0.05$ were considered to be statistically significant. 


\section{A outer segment-in (OS-in)}

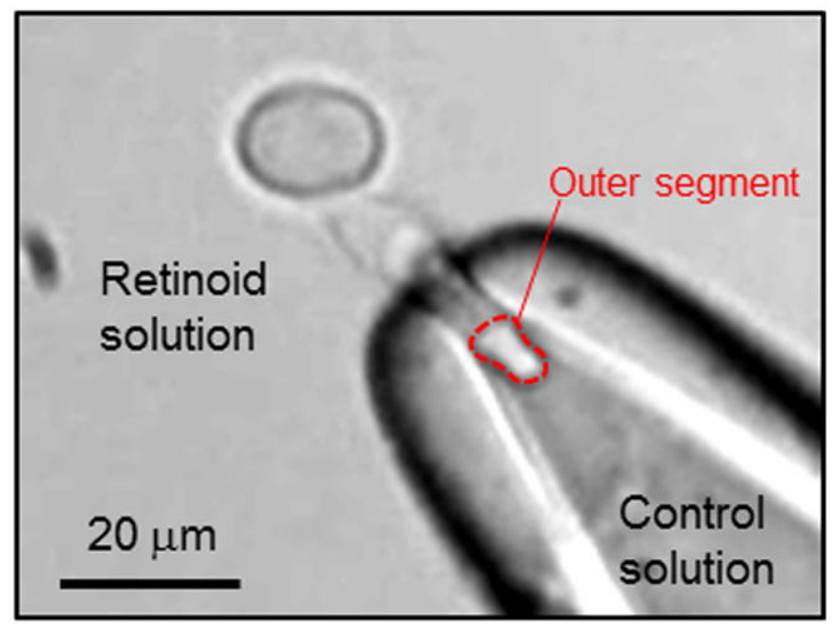

B

\section{9-cis retinal}
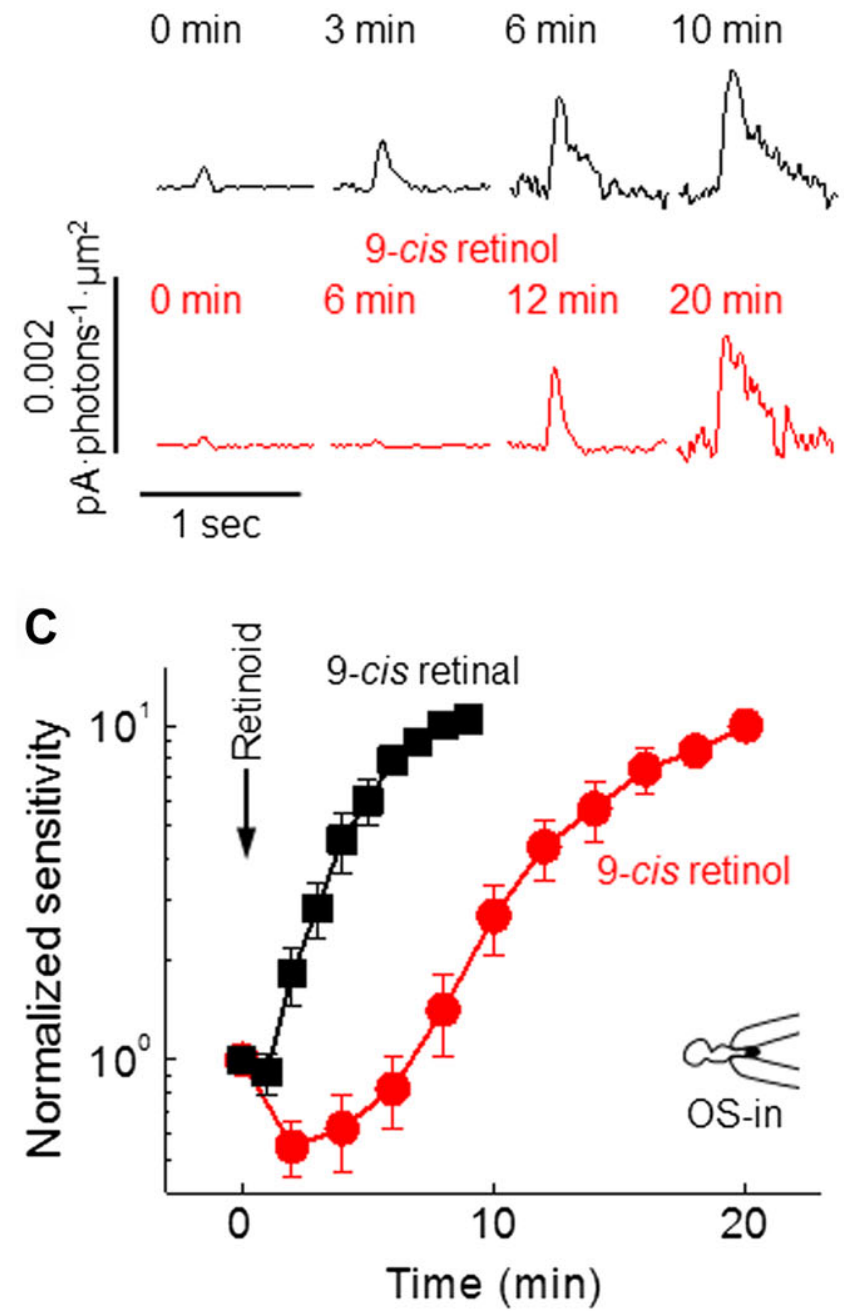

Fig. 1. Kinetics of red cone sensitivity recovery when exposing the inner segment to exogenous 9-cis chromophore. (A) Image of a red cone in the outer segment-in configuration under microscope with infrared illumination. (B) Dim flash responses from bleached red cones at indicated times after treatment with 9-cis retinal (black traces) and 9-cis retinol (red traces). Response amplitudes are normalized to the flash intensity (i.e. response

\section{Results}

Location of the cis retinol oxidation reaction in salamander red cones

In the living retina, fresh 11-cis retinol is provided by the Müller cells to cones, and ultimately used for pigment regeneration in the cone outer segments. However, as Müller cell processes do not make direct contact with the cone outer segments (Sarantis \& Mobbs, 1992; Garlipp \& Gonzalez-Fernandez, 2013), their 11-cis retinol is most likely delivered to the cone inner segments (Insinna et al., 2012). It is currently unclear whether the subsequent oxidation of 11-cis retinol to 11-cis retinal takes place in the inner or outer segments of cones. To determine the location of cis retinol oxidation in cones, we compared the time course of sensitivity recovery of fully bleached ( $>99 \%$ of visual pigment) isolated salamander red cones in a suction electrode when their outer $v s$. inner segment was exposed to 9-cis retinol, a commercially available more stable analogue of 11-cis retinol. As the suction electrode fits tightly around the photoreceptor cell, chromophore movement from the bath into the electrode was restricted so that only the part of the cell outside the electrode was exposed to it (Jin et al., 1994). Thus, we reasoned that exposing directly the part of the cell where oxidation takes place to 9-cis retinol would result in faster pigment regeneration compared to the reverse cell configuration. Isolated salamander red cones were drawn into the suction pipette from the outer segment to the mid-point of the inner segment (outer segment-in configuration; Fig. 1A) or from the cell body to the base of the outer segment (outer segment-out configuration; Fig. 2A). Cone pigments were fully bleached ( $>99 \%)$ by $505 \mathrm{~nm}$ light and then the cells were kept in darkness for 20 min until their sensitivity reached a steady state. At this point, 9-cis retinal or 9-cis retinol was added to the bath of the recording chamber and the change in cone sensitivity was monitored for the next $10 \mathrm{~min}$ for 9-cis retinal or $20 \mathrm{~min}$ for 9-cis retinol. Although the relation between the fraction of bleached pigment and sensitivity is nonlinear (Jones et al., 1993; Kefalov et al., 2005), physiological measurements of cone sensitivity provide a reliable method for monitoring the onset and kinetics of cone pigment regeneration. Thus, we used the kinetics of cone dark adaptation to estimate the rate of pigment regeneration in the outer segment, and the initial delay in this process to estimate the time required for the production/ delivery of 9-cis retinal to the outer segment.

We began by treating the inner segments of bleached red cones with 9-cis retinal in order to determine the kinetics of delivery of this retinoid to the outer segment and the subsequent rate of cone pigment regeneration in a configuration similar to that in vivo, with the retinoid delivered from the Müller cells. As 9-cis retinal can be used directly for pigment regeneration, the dark adaptation of cones in this case was rate-limited by its delivery to the outer segment. For outer segment-in cones (Fig. 1A), sensitivity recovery began shortly upon exposure to 9-cis retinal (Fig. 1B, black traces). The recovery onset was delayed by $\sim 1$ min (Fig. 1C, black filled squares), possibly reflecting the time required for 9-cis retinal taken up by the inner segment from the bath to reach the outer segment of the cell, sheltered inside the suction electrode. The subsequent dark adaptation of the cones proceeded rapidly and was largely completed within 5-6 min.

per photon) to allow direct comparison of sensitivity. (C) Sensitivity recovery time course of bleached red cones in the outer segment-in (OS-in) configuration incubated with 9-cis retinal (filled black squares; $n=10$ ) or 9-cis retinol (filled red circles; $n=8$ ). Data are shown as mean \pm SEM. 


\section{A outer segment-out (OS-out)}
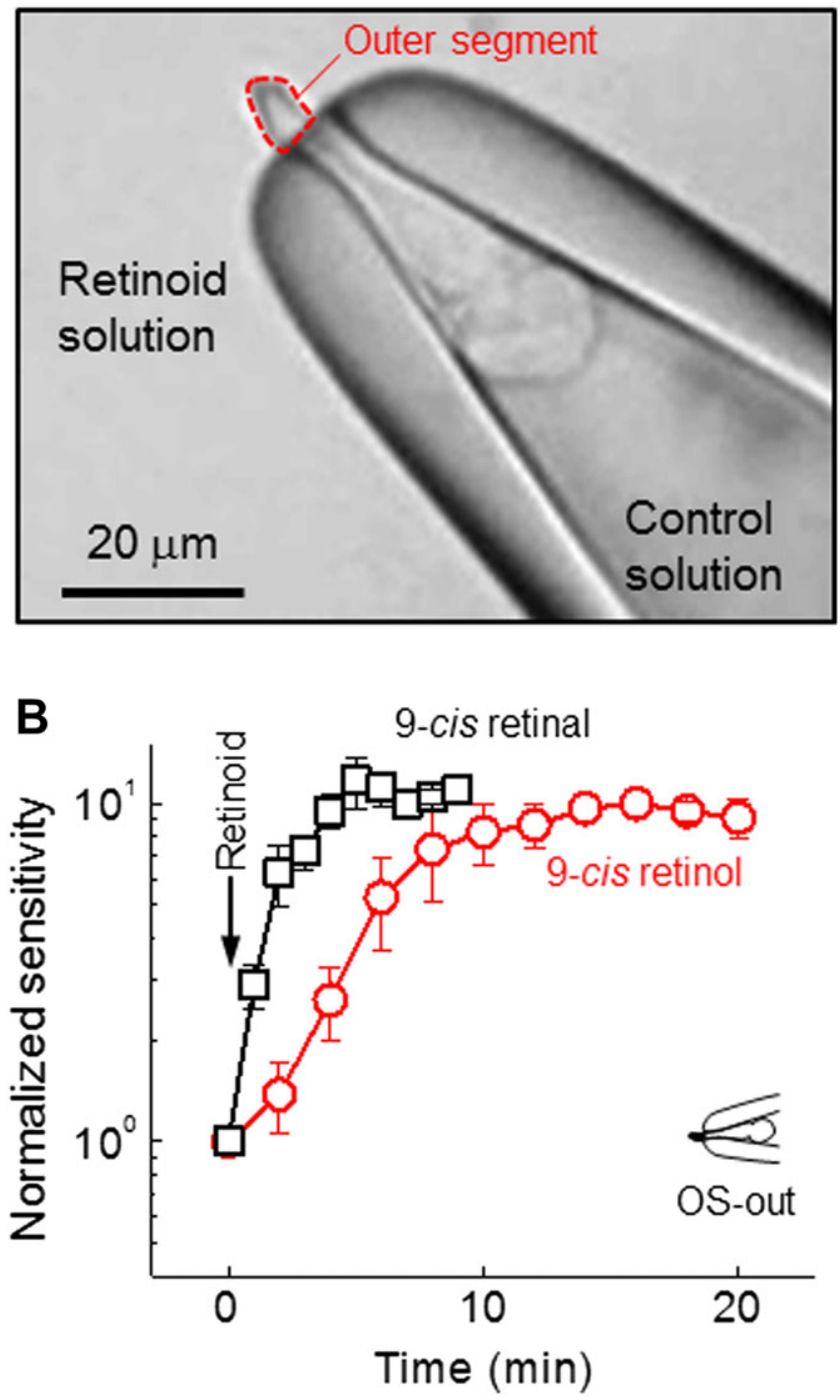

Fig. 2. Kinetics of red cone sensitivity recovery when exposing the outer segment to exogenous 9-cis chromophore. (A) Image of a red cone in the outer segment-out configuration under microscope with infrared illumination. (B) Sensitivity recovery time course of bleached red cones in the outer segment-out (OS-out) configuration incubated with 9-cis retinal (open black squares; $n=7$ ) or 9-cis retinol (open red circles; $n=8$ ). Data are shown as mean \pm SEM.

We next treat the inner segments of bleached cones with 9-cis retinol, a functional analogue to the 11-cis retinol produced by Müller cells in the retina (Sato \& Kefalov, 2016). In comparison with 9-cis retinal, use of 9-cis retinol requires the additional step of oxidation before it could be used for pigment regeneration. Thus, the overall time course of cone dark adaptation in this case was determined by two processes: production of 9-cis retinal and its delivery to the outer segment. As expected, the administration of 9-cis retinol to the inner segments resulted in gradual recovery of cone sensitivity, indicating that cones could oxidize 9-cis retinol and use it for pigment regeneration. However, this recovery was delayed by $\sim 6$ min (Fig. 1B, red traces; Fig. 1C, filled red circles) from the time of retinoid application. This delay in the onset of cone recovery with 9-cis retinol was substantially larger than the $\sim 1$ min delay observed for 9-cis retinal in the same configuration.
The slight transient decrease in sensitivity upon the application of 9-cis retinol was likely due to toxicity of its high concentration in the bath when the perfusion was stopped during retinoid application. Indeed, this decrease was not observed when the 9-cis retinol solution was diluted 10 -fold and the perfusion was not halted during its application, thus rapidly removing excess retinoid from the recording chamber (data not shown). The kinetics of the 9-cis retinol-driven recovery were also $>3$-fold slower than the recovery driven by 9-cis retinal so that cone dark adaptation was still incomplete 20 min after treatment. Thus, when the retinoid was applied to the cone inner segment, the recovery driven by 9-cis retinol was delayed by several min and proceeded $>3$-fold slower than the recovery driven by 9 -cis retinal. This could reflect the different traffic rates of 9-cis retinal and 9-cis retinol through the cone inner segment, or could be caused by the extra oxidation step of 9-cis retinol to 9-cis retinal. However, based only on the results above, we could not determine which of these two factors causes the delay or the slower recovery of cones when their inner segments were treated with 9-cis retinol vs. 9-cis retinal.

To address this question and distinguish between these two possibilities, we treated directly the outer segments of bleached cones with 9-cis retinal and 9-cis retinol by using the outer segment-out configuration (Fig. 2A). When applied directly to the outer segment, 9-cis retinal could be immediately taken up by the cone and began driving pigment regeneration and dark adaptation within seconds (Fig. 2B; black open squares). Direct comparison of cones treated with 9-cis retinal in the outer segment-in (Fig. 3A, open squares) and outer segment-out (Fig. 3A, filled squares) configurations demonstrated that their kinetics of dark adaptation were comparable. The only difference was the $\sim 1$ min delay in cone recovery when the chromophore was applied to the inner segment of the cones, compared to its direct application to the outer segment. This delay when 9-cis retinal is applied to the cone inner segment is comparable to that previously observed with 11-cis retinal (Jin et al., 1994) and likely reflects the time required for the retinoid to reach the outer segment. Notably, the ensuing 9-cis retinal-driven pigment regeneration in our experiments was independent of the site of retinoid application and completed within 5-6 min.

Having established the time course of dark adaptation driven by exogenous cis retinal in the two cell configurations, we next compared the kinetics of cone dark adaptation with cis retinol. Pigment regeneration in this case requires the additional step of oxidation to cis retinal before pigment regeneration. To investigate the subcellular localization and the kinetics of cis retinol oxidation in cones, we compared the kinetics of 9-cis retinol-driven cone dark adaptation in the outer-segment-in configuration with that of bleached cones in the outer segment-out configuration. Notably, similar to the treatment with 9-cis retinal in this configuration, and unlike the treatment with 9-cis retinol in the reverse configuration, the recovery of cone sensitivity in this case began immediately after the application of the retinoid (Fig. 2B, red open circles). Thus, cones could rapidly and efficiently utilize 9-cis retinol for robust pigment regeneration when the retinoid was applied directly to their outer segments. The kinetics of the 9-cis retinol-driven recovery of sensitivity were slower than those of the 9-cis retinal-driven recovery in this configuration (Fig. 2B), but comparable to those of the 9-cis retinol-driven recovery in the outer segment-in configuration (Fig. 3B). In both recording configurations, the kinetics of recovery with 9-cis retinol was $\sim 3$-fold slower than that with 9-cis retinal (Figs. 1C and 2B). Together, these results suggest that the delay in cone recovery when 9-cis retinol is applied to the inner segment is most likely due to the slow transport of this retinoid to the outer segment. The immediate 

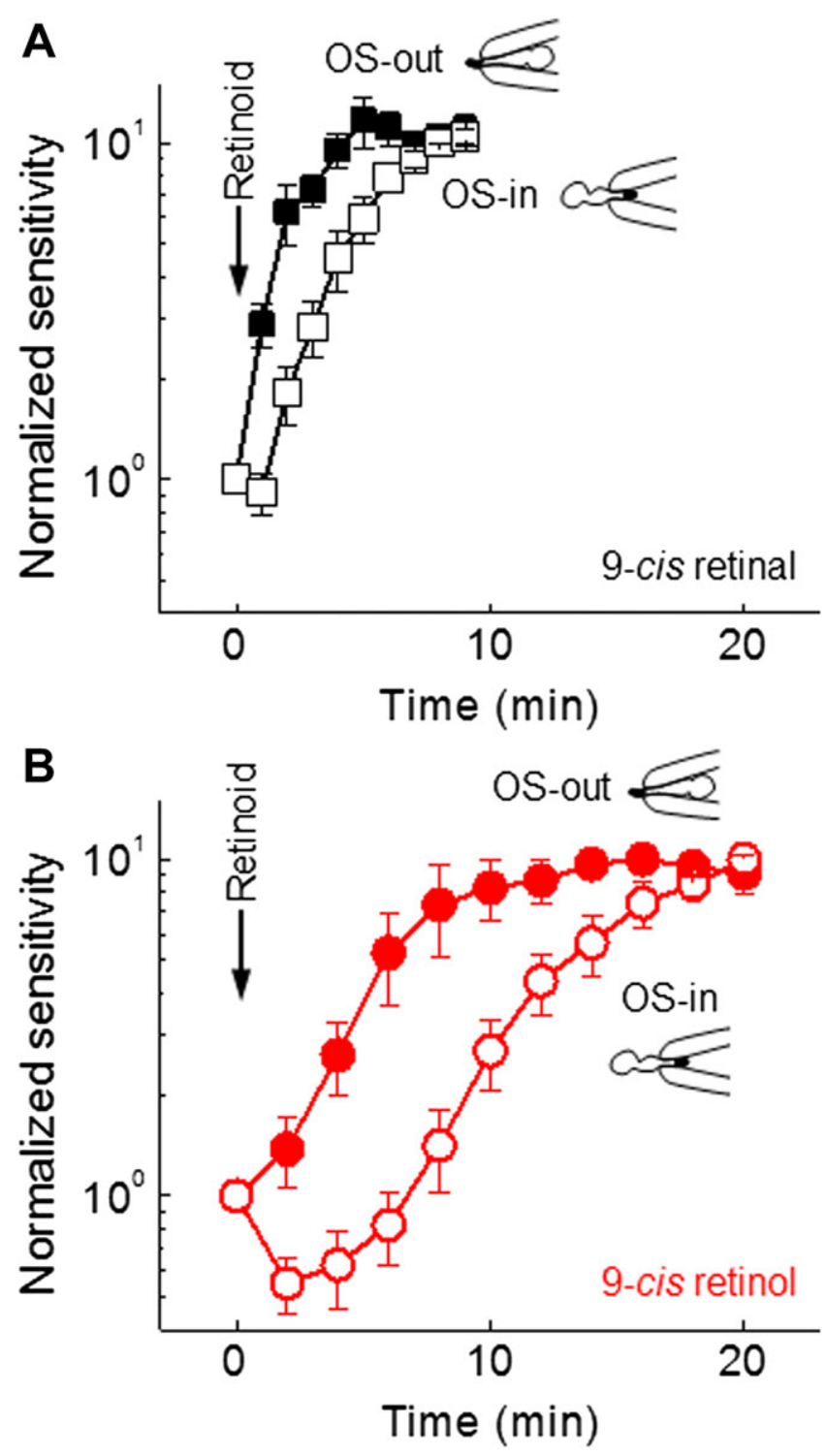

Fig. 3. Comparison of the kinetics of bleached red cone recovery in outer segment-in $v s$. outer segment-out configurations (data replotted from Fig. 1C and 2B). (A) Sensitivity recovery time course of bleached red cones incubated with 9-cis retinal in the OS-out (filled black squares) or OS-in (open black squares) configuration. (B) Sensitivity recovery time course of bleached red cones incubated with 9-cis retinol in the OS-out (filled red circles) or OS-in (open red circles) configuration.

onset of cone recovery when 9-cis retinol is applied directly to the outer segment is consistent with the notion that its oxidation to 9-cis retinal occurs in the outer segment itself rather than in the inner segment. The $\sim 3$-fold slower dark adaptation with 9-cis retinol over 9-cis retinal likely reflects the relatively slow oxidation of $c i s$ retinol to cis retinal in the outer segments of cones.

\section{cis Retinol oxidation in the truncated cone outer segment}

Our physiological data is consistent with the presence of cis retinol oxidation activity in cone outer segments. However, the spatial isolation between the outer and inner segments by our suction electrode was likely not ideal. Thus, the possibility still existed that in the outer segment-out configuration, 9-cis retinol from the bath could reach the tip of the inner segment and get oxidized there instead of in the outer segment. A second complication of the interpretation of our recordings arose from the slight decrease of cone sensitivity in the outer segment-in configuration upon the application of 9-cis retinol (Fig. 1C). This transient sensitivity suppression, which was not observed in the outer segment-out configuration, could also have masked any early onset of cone recovery mediated by oxidation in the inner segment. To rule out these possibilities, we tested directly the ability of truncated salamander cone outer segments to oxidize 9-cis retinol and use it for pigment regeneration. As suction recordings from truncated cone outer segments are quite challenging, we used microspectrophotometry (MSP) to measure directly pigment regeneration in 9-cis retinol-treated cone outer segments broken off from the rest of the cell. Truncated outer segments from dark-adapted red cones were identified by their shape and their characteristic absorption spectrum (Fig. 4A) with a peak at $610 \pm 4 \mathrm{~nm}$ (mean \pm S.D., $n=4)$, reflecting the mix of 11-cis $\mathrm{A}_{1}$ and 11-cis $\mathrm{A}_{2}$ chromophore in the retina of larval tiger salamanders (Makino et al., 1999). The outer segments were then bleached by bright light, which, as expected, resulted in the ablation of the pigment peak in the absorption spectrum (Fig. 4B). Critically, treatment of such bleached truncated cone outer segments with 9-cis retinol resulted in robust 9-cis pigment regeneration as demonstrated by the reappearance of a blue-shifted pigment spectrum (Fig. 4C) with a peak at $544 \pm 9 \mathrm{~nm}$ (mean \pm S.D., $n=4)$. This peak wavelength is comparable to the reported value for the 9-cis pigment in salamander red cones (542 nm) (Makino et al., 1999). The absorption peak disappeared after an additional bright light illumination, confirming that it originated from bleachable pigment and not from nonspecific retinoid products. This result clearly demonstrates that 9-cis retinol can be oxidized in the truncated cone outer segment and used for 9-cis pigment formation. As a control, the same experiments were done with intact salamander red rods (Fig. 4E) following a bleach (Fig. 4F). In this case, pigment regeneration was not observed after 9-cis retinol treatment (Fig. 4G). However, subsequent treatment with 11-cis retinal produced robust pigment regeneration (Fig. $4 \mathrm{H}$ ). The inability of salamander rods to utilize 9-cis retinol for pigment regeneration is consistent with our recent finding that 9-cis retinol oxidation activity is cone-specific (Sato \& Kefalov, 2016). Importantly, the lack of regeneration in rods under these conditions also ruled out contamination with 9-cis retinal in our 9-cis retinol preparation. Together, these MSP results substantiate our findings from single-cell recordings above and clearly demonstrate that the oxidation of 9-cis retinol takes place in cone outer segments and can drive robust cone pigment regeneration.

\section{Discussion}

\section{Oxidation and traffic of 9-cis retinol in cones}

This study builds on two elegant previous studies that showed that cis retinol can drive cone pigment regeneration (Jones et al., 1989) and that the movement of retinoid from the inner to outer segment of salamander cones can be studied by restricting access with a suction electrode (Jin et al., 1994). Here, we used a similar approach to determine the cone compartment where oxidation of cis retinol takes place. We found that when the cone outer segment was treated with 9-cis retinal, dark adaptation began essentially immediately (Fig. 2B). When the cone inner segment was treated with 9-cis retinal, the recovery onset was delayed by $\sim 1$ min (Fig. 1C) reflecting the time required for the movement of 9-cis retinal to the outer segment before pigment regeneration can begin. The kinetics 

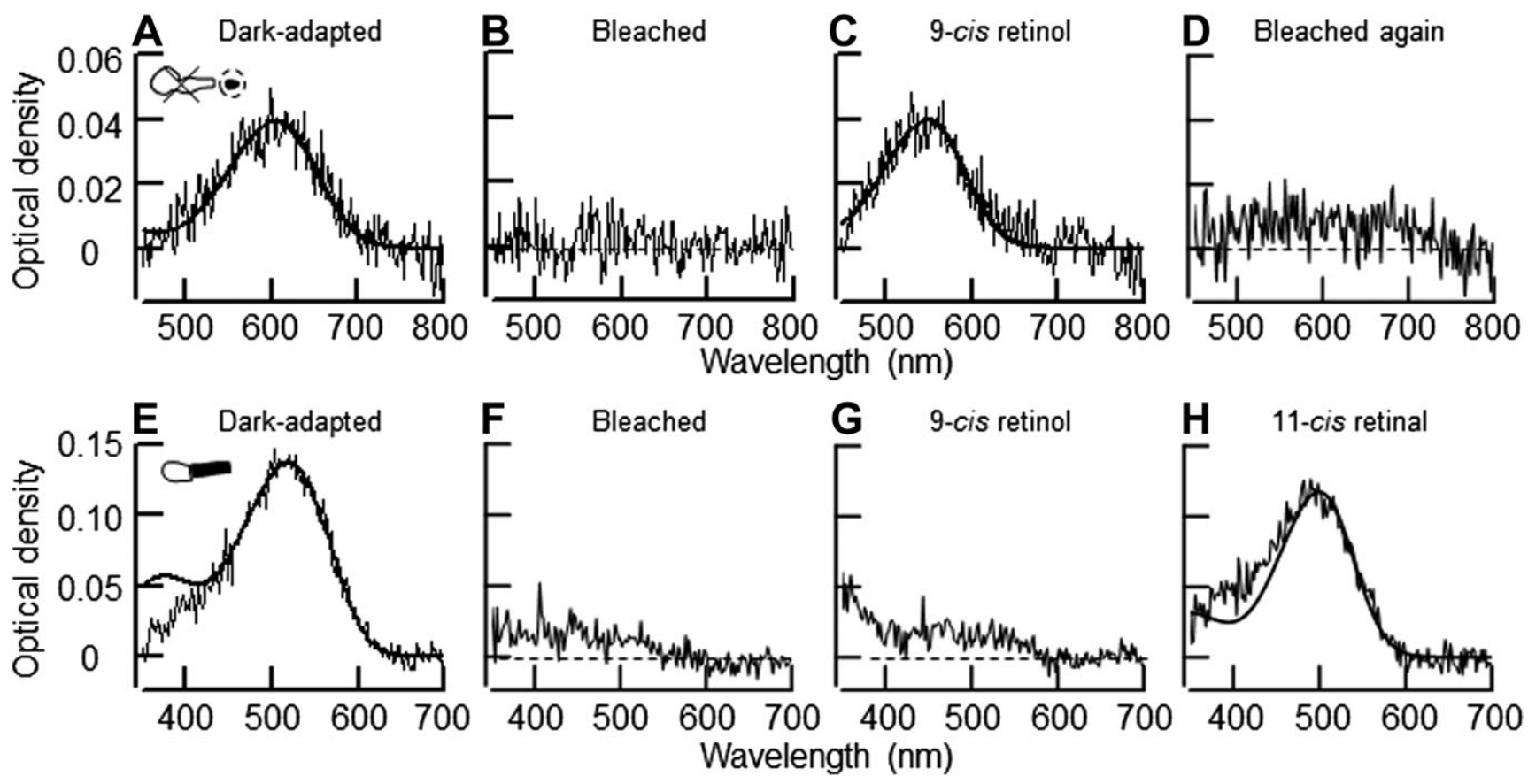

Fig. 4. Microspectrophotometric measurement of pigment regeneration in a truncated cone outer segment and in an intact rod. (A) Absorbance spectrum of a dark-adapted cone outer segment. Fitted to the data is a visual pigment template with peak wavelength at $605 \mathrm{~nm}$ and an optical density of 0.039. (B) Absorbance spectrum of the same cone outer segment as in (A) recorded after $>99 \%$ of the visual pigment had been bleached. $(\mathbf{C})$ Absorbance spectrum of the same bleached cone outer segment as in $(\mathbf{B})$ recorded after treatment with 9-cis retinol. Fitted to the data is a visual pigment template with peak wavelength at $549 \mathrm{~nm}$ and an optical density of 0.040 . (D) Absorbance spectrum of the same regenerated cone outer segment as in (C) recorded after a second $>99 \%$ bleach of its visual pigment. (E) Absorbance spectrum of the outer segment of an intact dark-adapted red rod. Fitted to the data is a visual pigment template with peak wavelength at $518 \mathrm{~nm}$ and an optical density of 0.14. (F) Absorbance spectrum of the same rod as in (E) recorded after $>99 \%$ of the visual pigment had been bleached. $(\mathbf{G})$ Absorbance spectrum of the same bleached rod as in (F) recorded after treatment with 9-cis retinol. (H) Absorbance spectrum of the same bleached rod as in $(\mathbf{G})$ recorded after treatment with 11-cis retinal. Fitted to the data is a visual pigment template with peak wavelength at $500 \mathrm{~nm}$ and an optical density of 0.12 .

of the subsequent dark adaptation was identical in the two configurations (Fig. 3A). Notably, after its onset, the kinetics of cone recovery with 9-cis retinol in the two configurations were also identical (Fig. 3B) but $\sim 3$-fold slower than the recovery kinetics with 9-cis retinal. This result suggests that the oxidation of cis retinol proceeds relatively slowly and might be rate-limiting for the regeneration of pigment via the retina visual cycle. On the other hand, after exposing the inner segment of bleached cones to 9-cis retinol, sensitivity recovery onset was delayed by $\sim 6 \mathrm{~min}$. The absence of such delay in the outer segment-out configuration (Fig. 2B) allowed us to rule out slow 9-cis retinol oxidation as its source. Instead, this result suggested that this delay likely reflects the time required for 9-cis retinol to travel from the inner to the outer segments of cones, similar to the case of 9-cis retinal. Thus, the most likely explanation for our electrophysiological results is that 9-cis retinol is oxidized in the cone outer segment.

Notably, the delay in recovery onset observed in outer segment-in cones with 9-cis retinol (Fig. 1C) was similar to the delay observed in intact salamander retina [Fig. 5A in (Wang et al., 2009)]. Thus, even though isomeric differences between endogenous 11-cis retinoids and their exogenous 9-cis analogs used in this study could exist, they do not appear to affect dramatically cone dark adaptation. Together, our results suggest that in the intact eye, cis retinol is transported from the microvilli of Müller cells to cone outer segments via the inner segments and is then oxidized to the cis retinal needed for pigment regeneration. The substantially longer time for 9-cis retinol delivery from the inner to the outer segment compared to 9-cis retinal (Fig. 1C) suggests the presence of an efficient mechanism for transporting cis retinal from cone inner segment to the outer segment. Alternatively, the movement of cis retinol through the cone inner segment could somehow be obstructed. Photoreceptors are thought not to have retinoid binding proteins that regulate the transport of hydrophobic retinoids within the cytosol. Indeed, cellular retinal binding protein (Collery et al., 2008) and cellular retinol binding protein (Bok et al., 1984) have not been detected from photoreceptors. Thus, chromophore-binding proteins are not likely to be involved in the regulation of retinoid transport through the cone inner segment. Finally, it is also worth pointing out that due to their different chemical nature, retinol is less hydrophobic than retinal, and would therefore be expected to partition and diffuse differently through the cellular membranes and cytosol of cone photoreceptors.

\section{cis Retinol oxidase in cone outer segment}

Our MSP data (Fig. 4) clearly demonstrate the presence of cis retinol oxidase in the outer segments of cones. This result is consistent with the hypothesis that RDH8 is the enzyme responsible for this reaction. RDH8 is expressed in cone outer segments (Maeda et al., 2005; Miyazono et al., 2008) and shows weak but detectable activity to cis retinoid (Rattner et al., 2000). In addition, deletion of RDH8 blocks the sensitivity recovery with exogenous 9-cis retinol in mouse M-cones (Kolesnikov et al., 2015). However, RDH8 is expressed not only in cones but also in rods (Maeda et al., 2005; 
Miyazono et al., 2008), a distribution inconsistent with the conespecific oxidation of cis retinol. One possibility is that RDH8 is not itself the cis retinol dehydrogenase, but rather it supports this reaction by providing $\mathrm{NADP}^{+}$through the reduction of all-trans retinal (Kaylor et al., 2014). Further studies will be needed to determine the exact involvement of RDH8 in the retina visual cycle.

In contrast to our finding from salamander, a recent biochemical study reported cis-retinol oxidation activity in the carp cone inner segments but not in the outer segments (Sato et al., 2013; Sato et al., 2015). RDH13L, the enzyme responsible for this inner segment activity, is localized in the inner segments of carp cones but it is not found in the amphibian genome. Notably, a functional homologue of RDH13L, RDH14, is expressed in rod and cone outer segments in bovine retina (Haeseleer et al., 2002). However, the functional role of RDH14 in the retina visual cycle, and specifically in the oxidation of 11-cis retinol, is still unknown. The conflicting results obtained from salamander cones (this study) and from carp cones (Sato et al., 2013; Sato et al., 2015) may simply reflect interspecies difference. Alternatively, co-factors or other reaction modifiers could be required for the efficient oxidation of cis retinol in cones. In that case, their availability or absence in different experimental conditions could determine the subcellular location of this reaction. Similarly, the presence or absence of cellular compartmentalization in different experimental conditions could also affect the efficiency of cis retinol oxidation. Nevertheless, the robust pigment regeneration and dark adaptation observed in our experiments with cone outer segment-delivered 9-cis retinol clearly demonstrate the presence of cis retinol dehydrogenase activity in the outer segments of cones but not rods.

Finally, it is also possible that another enzyme, different from RDH8 and RDH14, is responsible for the oxidation of cis retinol in the outer segments of cones. Notably, RPE65, the isomerohydrolase of the canonical RPE visual cycle, was recently shown to be expressed in the outer segments of cones in some mouse strains (Tang et al., 2011b) as well as in humans (Tang et al., 2011a). It has been suggested that this protein might be involved in the cone visual cycle by acting as a retinol-binding protein (Tang et al., 2013). An interesting possibility is that RPE65 might interact with the conespecific RDH and, in that way, facilitate the oxidation of cis retinol in cones. However, the functional role of RPE65 in cones is still unclear and its possible involvement in the retina visual cycle has not been explored.

\section{Co-factor driving the cis retinol oxidation in cones}

Previous studies with amphibian rods (Kawamura \& Murakami, 1989) and fish rods and cones (Takemoto et al., 2009) both suggest that the inside of the truncated outer segment remains accessible to bath-applied compounds. Thus, the truncated outer segments in our experiments also likely remained open, allowing wash out of small molecules from the cytoplasm. $\mathrm{NADP}^{+}$is thought to be a cofactor in the cone-specific cis retinol oxidation (Mata et al., 2002). However, we observed 9-cis retinol-driven pigment regeneration in the truncated cone outer segment (Fig. 4) even though $\mathrm{NADP}^{+}$was expected to be washed out rapidly. Thus, the cofactor for $c i s$ retinol oxidation in the truncated cone outer segment is not likely to be $\mathrm{NADP}^{+}$. A possible alternative co-factor is all-trans retinal, which is insoluble and shown to work as a co-factor in the cis retinol oxidation reaction by carp RDH13L and mouse RDH14 (Sato et al., 2015). All-trans retinal is released at a high concentration (up to $3 \mathrm{mM}$ ) in cone outer segments following the bleaching of their visual pigments (Harosi, 1975). In the intact cones, all-trans retinal is then rapidly converted to all-trans retinol by NADPH-driven reduction (Ala-Laurila et al., 2006). However, our attempt to detect acceleration of cis retinol oxidation by all-trans retinal was not successful: addition of all-trans retinal to the 9-cis retinol treatment of either outer segment-in (Fig. 1A) or outer segment-out (Fig. 2A) bleached cones did not affect the kinetics of cone dark adaptation (data not shown). Thus, the cofactor for cis-retinol oxidation is still unclear and further experiments will be needed to clarify the molecular mechanism of this key reaction of the retina visual cycle. Our finding that the oxidation of cis retinol takes place in the outer segments of cones should inform future studies and help in the eventual identification of the enzyme responsible for this reaction.

\section{Author contribution}

SS and VJK designed the study and wrote the paper. SS and VJK designed, performed, and analyzed the experiments shown in Figs. 1, 2, and 3. RF and MCC designed, performed, and analyzed the experiments shown in Fig. 4. All authors reviewed the results and approved the final version of the manuscript.

\section{Acknowledgments}

This work was supported by National Institutes of Health (NIH) grants EY019312 and EY025696 (V.J.K.), EY001157 (M.C.C.), EY002687 to the Department of Ophthalmology and Visual Sciences at Washington University, and by Research to Prevent Blindness. We are grateful to the National Eye Institute and Rosalie Crouch from the Medical University of South Carolina for the 11-cis retinal.

\section{References}

Ala-Laurila, P., Cornwall, M.C., Crouch, R.K. \& Kono, M. (2009). The action of 11-cis-retinol on cone opsins and intact cone photoreceptors. Journal of Biological Chemistry 284, 16492-16500.

Ala-laurila, P., Kolesnikov, A.V., Crouch, R.K., Tsina, E., Shukolyukov, S.A., Govardovskit, V.I., Koutalos, Y., Wiggert, B., Estevez, M.E. \& Cornwall, M.C. (2006). Visual cycle: Dependence of retinol production and removal on photoproduct decay and cell morphology. Journal of General Physiology 128, 153-169.

Bok, D., Ong, D.E. \& Chytil, F. (1984). Immunocytochemical localization of cellular retinol binding protein in the rat retina. Investigative Ophthalmology \& Visual Science 25, 877-883.

Collery, R., Mcloughlin, S., Vendrell, V., Finnegan, J., Crabb, J.W., SAari, J.C. \& Kennedy, B.N. (2008). Duplication and divergence of zebrafish CRALBP genes uncovers novel role for RPE- and MullerCRALBP in cone vision. Investigative Ophthalmology \& Visual Science 49, 3812-3820.

Ebrey, T. \& Koutalos, Y. (2001). Vertebrate photoreceptors. Progress in Retinal and Eye Research 20, 49-94.

Frederiksen, R., Boyer, N.P., Nickle, B., Chakrabarti, K.S., Koutalos, Y., Crouch, R.K., Oprian, D. \& Cornwall, M.C. (2012). Low aqueous solubility of 11-cis-retinal limits the rate of pigment formation and dark adaptation in salamander rods. Journal of General Physiology 139, 493-505.

Garlipp, M.A. \& Gonzalez-Fernandez, F. (2013). Cone outer segment and Muller microvilli pericellular matrices provide binding domains for interphotoreceptor retinoid-binding protein (IRBP). Exp. Eye Res. 113, 192-202.

Haeseleer, F., Jang, G.F., Imanishi, Y., Driessen, C.A., Matsumura, M., Nelson, P.S. \& PalczewsKi, K. (2002). Dual-substrate specificity short chain retinol dehydrogenases from the vertebrate retina. Journal of Biological Chemistry 277, 45537-45546.

Harosi, F.I. (1975). Absorption spectra and linear dichroism of some amphibian photoreceptors. Journal of General Physiology 66, 357-382. 
Iмамото, Y. \& Shichida, Y. (2014). Cone visual pigments. Biochimica et Biophysica Acta 1837, 664-673.

Insinna, C., Daniele, L.L., Davis, J.A., Larsen, D.D., Kuemmel, C., Wang, J., Nikonov, S.S., Knox, B.E. \& Pugh, E.N., Jr. (2012). An S-opsin knock-in mouse (F81Y) reveals a role for the native ligand 11-cis-retinal in cone opsin biosynthesis. Journal of Neuroscience $\mathbf{3 2}$, 8094-8104.

Jin, J., Jones, G.J. \& CoRnwall, M.C. (1994). Movement of retinal along cone and rod photoreceptors. Visual Neuroscience 11, 389-399.

Jones, G.J., Crouch, R.K., Wiggert, B., Cornwall, M.C. \& Chader, G.J. (1989). Retinoid requirements for recovery of sensitivity after visual-pigment bleaching in isolated photoreceptors. Proceedings of the National Academy of Sciences of the United States of America $\mathbf{8 6}$ 9606-9610.

Jones, G.J., Fein, A., MacNichol, E.F., Jr. \& Cornwall, M.C. (1993). Visual pigment bleaching in isolated salamander retinal cones. Microspectrophotometry and light adaptation. Journal of General Physiology 102, 483-502.

Kanan, Y., Kasus-Jacobi, A., Moiseyev, G., Sawyer, K., Ma, J.X. \& AL-Ubaidi, M.R. (2008). Retinoid processing in cone and Muller cell lines. Experimental Eye Research 86, 344-354.

Kawamura, S. \& Murakami, M. (1989). Regulation of cGMP levels by guanylate cyclase in truncated frog rod outer segments. Journal of General Physiology 94, 649-668.

KaYlor, J.J., Cook, J.D., Makshanoff, J., Bischoff, N., Yong, J. \& Travis, G.H. (2014). Identification of the 11-cis-specific retinyl-ester synthase in retinal Muller cells as multifunctional $O$-acyltransferase (MFAT). Proceedings of the National Academy of Sciences of the United States of America 111, 7302-7307.

KaYlor, J.J., Yuan, Q., Cook, J., Sarfare, S., Makshanoff, J., Miu, A., Kim, A., Kim, P., Habib, S., Roybal, C.N., Xu, T., Nusinowitz, S. \& Travis, G.H. (2013). Identification of DES1 as a vitamin A isomerase in Muller glial cells of the retina. Nature Chemical Biology 9, 30-36.

Kefalov, V.J., Crouch, R.K. \& Cornwall, M.C. (2001). Role of noncovalent binding of 11-cis-retinal to opsin in dark adaptation of rod and cone photoreceptors. Neuron 29, 749-755.

Kefalov, V.J., Estevez, M.E., Kono, M., Goletz, P.W., Crouch, R.K., Cornwall, M.C. \& YaU, K.W. (2005). Breaking the covalent bond-A pigment property that contributes to desensitization in cones. Neuron 46, 879-890.

Kolesnikov, A.V., Maeda, A., Tang, P.H., Imanishi, Y., Palczewski, K. \& Kefalov, V.J. (2015). Retinol dehydrogenase 8 and ATP-binding cassette transporter 4 modulate dark adaptation of M-cones in mammalian retina. Journal of Physiology 593, 4923-4941.

Kolesnikov, A.V., Tang, P.H., Parker, R.O., Crouch, R.K. \& Kefalov, V.J. (2011). The mammalian cone visual cycle promotes rapid M/L-cone pigment regeneration independently of the interphotoreceptor retinoidbinding protein. Journal of Neuroscience 31, 7900-7909.

Koutalos, Y. \& CoRnWALl, M.C. (2010). Microfluorometric measurement of the formation of all-trans-retinol in the outer segments of single isolated vertebrate photoreceptors. Methods in Molecular Biology 652, 129-147.

Maeda, A., Maeda, T., Imanishi, Y., Kuksa, V., Alekseev, A., Bronson, J.D., Zhang, H., Zhu, L., Sun, W., Saperstein, D.A., Rieke, F., BAeHr, W. \& PalcZewski, K. (2005). Role of photoreceptor-specific retinol dehydrogenase in the retinoid cycle in vivo. Journal of Biological Chemistry 280, 18822-18832.

MaKino, C.L. \& DodD, R.L. (1996). Multiple visual pigments in a photoreceptor of the salamander retina. Journal of General Physiology 108, 27-34.

Makino, C.L., Groesbeek, M., Lugtenburg, J. \& Baylor, D.A. (1999). Spectral tuning in salamander visual pigments studied with dihydroretinal chromophores. Biophysical Journal 77, 1024-1035.
Mata, N.L., Radu, R.A., Clemmons, R.C. \& Travis, G.H. (2002), Isomerization and oxidation of vitamin $\mathrm{A}$ in cone-dominant retinas: A novel pathway for visual-pigment regeneration in daylight. Neuron 36, 69-80.

Miyazono, S., Shimauchi-Matsukawa, Y., Tachibanaki, S. \& KaWAmura, S. (2008). Highly efficient retinal metabolism in cones. Proceedings of the National Academy of Sciences of the United States of America 105, 16051-16056.

Rattner, A., Smallwood, P.M. \& Nathans, J. (2000). Identification and characterization of all-trans-retinol dehydrogenase from photoreceptor outer segments, the visual cycle enzyme that reduces alltrans-retinal to all-trans-retinol. Journal of Biological Chemistry $\mathbf{2 7 5}$, 11034-11043.

SAARI, J.C. (2012). Vitamin A metabolism in rod and cone visual cycles. Annual Review of Nutrition 32, 125-145.

SARANTIS, M. \& MobBs, P. (1992). The spatial relationship between Muller cell processes and the photoreceptor output synapse. Brain Research 584, 299-304.

Sato, S., Fukagawa, T., Tachibanaki, S., Yamano, Y., Wada, A. \& KAWAMURA, S. (2013). Substrate specificity and subcellular localization of the aldehyde-alcohol redox-coupling reaction in carp cones. Journal of Biological Chemistry 288, 36589-36597.

Sato, S. \& Kefalov, V.J. (2016). cis Retinol oxidation regulates photoreceptor access to the retina visual cycle and cone pigment regeneration. Journal of Physiology 594, 6753-6765.

Sato, S., Miyazono, S., Tachibanaki, S. \& Kawamura, S. (2015) RDH13L, an enzyme responsible for the aldehyde-alcohol redox coupling reaction (AL-OL coupling reaction) to supply 11-cis retinal in the carp cone retinoid cycle. Journal of Biological Chemistry 290 , 2983-2992.

Sherry, D.M., Bui, D.D. \& Degrip, W.J. (1998). Identification and distribution of photoreceptor subtypes in the neotenic tiger salamander retina. Visual Neuroscience 15, 1175-1187.

Takemoto, N., Tachibanaki, S. \& Kawamura, S. (2009). High cGMP synthetic activity in carp cones. Proceedings of the National Academy of Sciences of the United States of America 106, 11788-11793.

TANG, P.H., Buhusi, M.C., MA, J.X. \& CROUCH, R.K. (2011a). RPE65 is present in human green/red cones and promotes photopigment regeneration in an in vitro cone cell model. Journal of Neuroscience 31 , 18618-18626.

Tang, P.H., Kono, M., Koutalos, Y., Ablonczy, Z. \& Crouch, R.K. (2013). New insights into retinoid metabolism and cycling within the retina. Progress in Retinal and Eye Research 32, 48-63.

TANG, P.H., Wheless, L. \& Crouch, R.K. (2011b). Regeneration of photopigment is enhanced in mouse cone photoreceptors expressing RPE65 protein. Journal of Neuroscience 31, 10403-10411.

Wang, J.S., Estevez, M.E., Cornwall, M.C. \& Kefalov, V.J. (2009). Intra-retinal visual cycle required for rapid and complete cone dark adaptation. Nature Neuroscience 12, 295-302.

Wang, J.S. \& Kefalov, V.J. (2011). The cone-specific visual cycle. Progress in Retinal and Eye Research 30, 115-128.

Wang, J.S., Nymark, S., Frederiksen, R., Estevez, M.E., Shen, S.Q., Corbo, J.C., Cornwall, M.C. \& Kefalov, V.J. (2014). Chromophore supply rate-limits mammalian photoreceptor dark adaptation. Journal of Neuroscience 34, 11212-11221.

Wright, C.B., Redmond, T.M. \& NicKerson, J.M. (2015). A history of the classical visual cycle. Progress in Molecular Biology and Translational Science 134, 433-448.

Xue, Y., Shen, S.Q., Jui, J., Rupp, A.C., Byrne, L.C., Hattar, S., Flannery, J.G., Corbo, J.C. \& Kefalov, V.J. (2015). CRALBP supports the mammalian retinal visual cycle and cone vision. Journal of Clinical Investigation 125, 727-738. 